\title{
Supramolecular Hydrogels via Light-Responsive Homoternary Cross-links
}

\author{
Lei Zou, ${ }^{1}$ Christopher J. Addonizio, ${ }^{1}$ Bo Su,${ }^{1}$ Matthew J. Sis, ${ }^{1}$ Adam S. Braegelman, ${ }^{1}$ \\ Dongping Liu, ${ }^{l}$ and Matthew J. Webber ${ }^{1, *}$
}

1- University of Notre Dame, Department of Chemical \& Biomolecular Engineering, Notre Dame, IN 46556 USA

[*] Person to whom correspondences should be addressed:

Prof. Matthew J. Webber

University of Notre Dame

Department of Chemical \& Biomolecular Engineering

Notre Dame, IN 46556 USA

mwebber@nd.edu

\section{Contents of Supporting Information:}

Figure S1- ${ }^{1} \mathrm{H}-\mathrm{NMR}$ of AzoSM precursors

Figure S2- Supplemental ITC data for CB[8]-AzoSM peformed at $4{ }^{\circ} \mathrm{C}$ and $37^{\circ} \mathrm{C}$.

Figure S3- Picture of NMR tube containing cis-AzoSM and CB[8] precipitate

Figure S4- ${ }^{1} \mathrm{H}-\mathrm{NMR}$ exploring thermal cis-to-trans relaxation for AzoSM w/ CB[8]

Figure S5- UV-vis for cis-to-trans relaxation for AzoSM w/ and w/o CB[8]

Figure S6- SEC trace of synthesized pNIPAM $_{\mathrm{x}}$-r-pDMAEA $\mathrm{p}_{1}$ copolymers

Figure $\mathbf{S 7}$ - ${ }^{1} \mathrm{H}-\mathrm{NMR}$ of synthesized pNIPAM $\mathrm{x}_{\mathrm{x}}$-r-pDMAEA ${ }_{1}$ copolymers

Figure S8- ${ }^{1} \mathrm{H}-\mathrm{NMR}$ for azobenzene modification of $\mathrm{pNIPAM}_{\mathrm{x}}$-r-pDMAEA ${ }_{1}$ copolymers

Figure S9- Gross assessment of LCST for polymers before and after azobenzene modification

Figure S10- Photograph of CB[8] insolubility in pNIPAM ${ }_{10}-r-p D M A E A_{1}$

Figure S11- Frequency-dependent complex viscosity of pNIPAM ${ }_{15}$-r-pDMAEA(Azo) ${ }_{1}$

Figure S12- Supplemental rheology for other copolymer ratios

Figure S13- Hydrogel swelling and dissolution into an $\mathrm{H} 2 \mathrm{O}$ phase

Figure S14- UV-vis for cis-to-trans relaxation of pNIPAM $15-\mathrm{r}-\mathrm{pDMAEA}(\mathrm{Azo})_{1} \mathrm{w} /$ \& w/o CB[8]

Also available online:

Movie S1: Thermal relaxation and hydrogel self-healing 


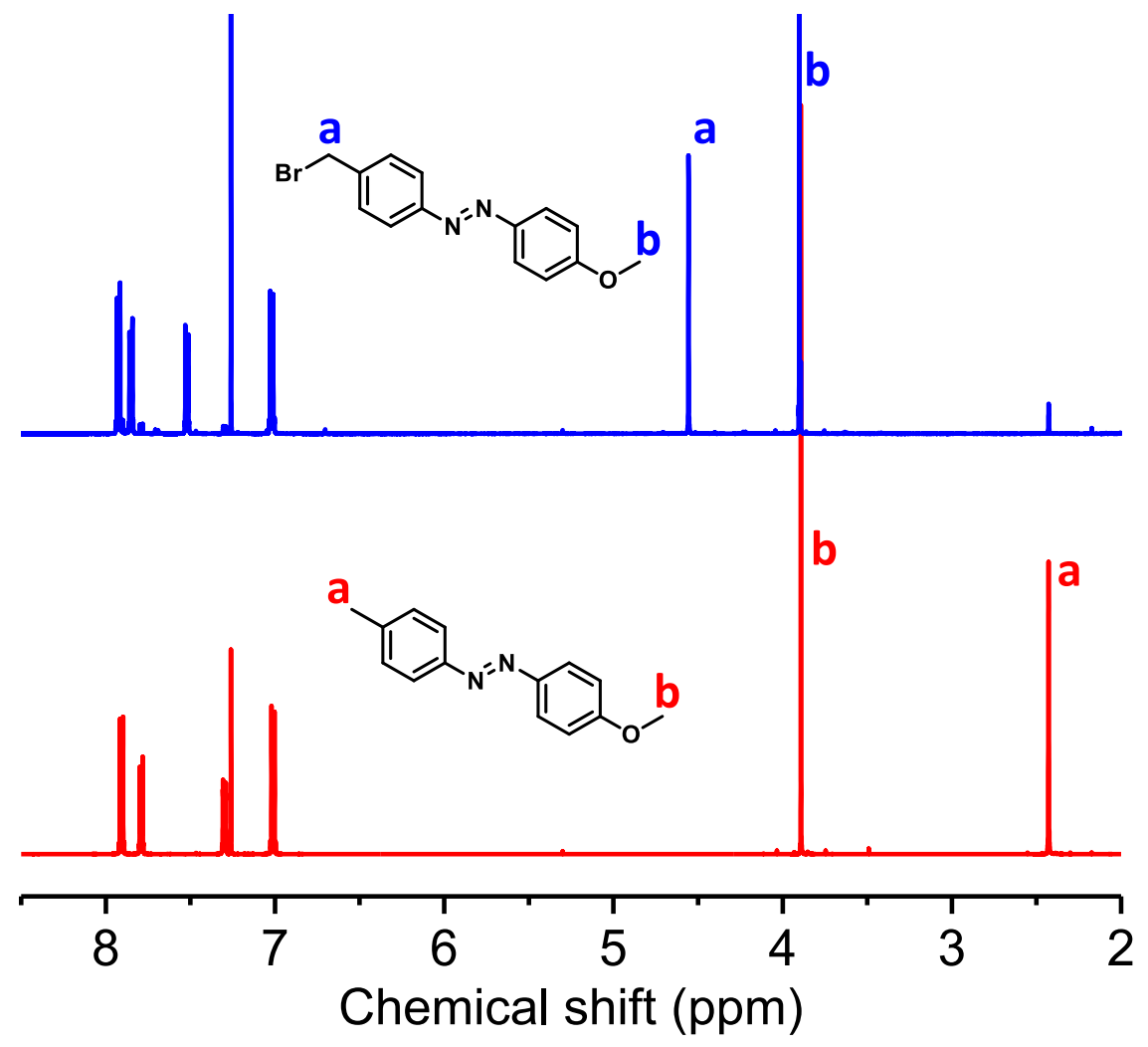

Figure S1: ${ }^{1} H$-NMR spectra for precursors prepared in the synthesis of AzoSM, specifically AzoBr used for polymer modification. 

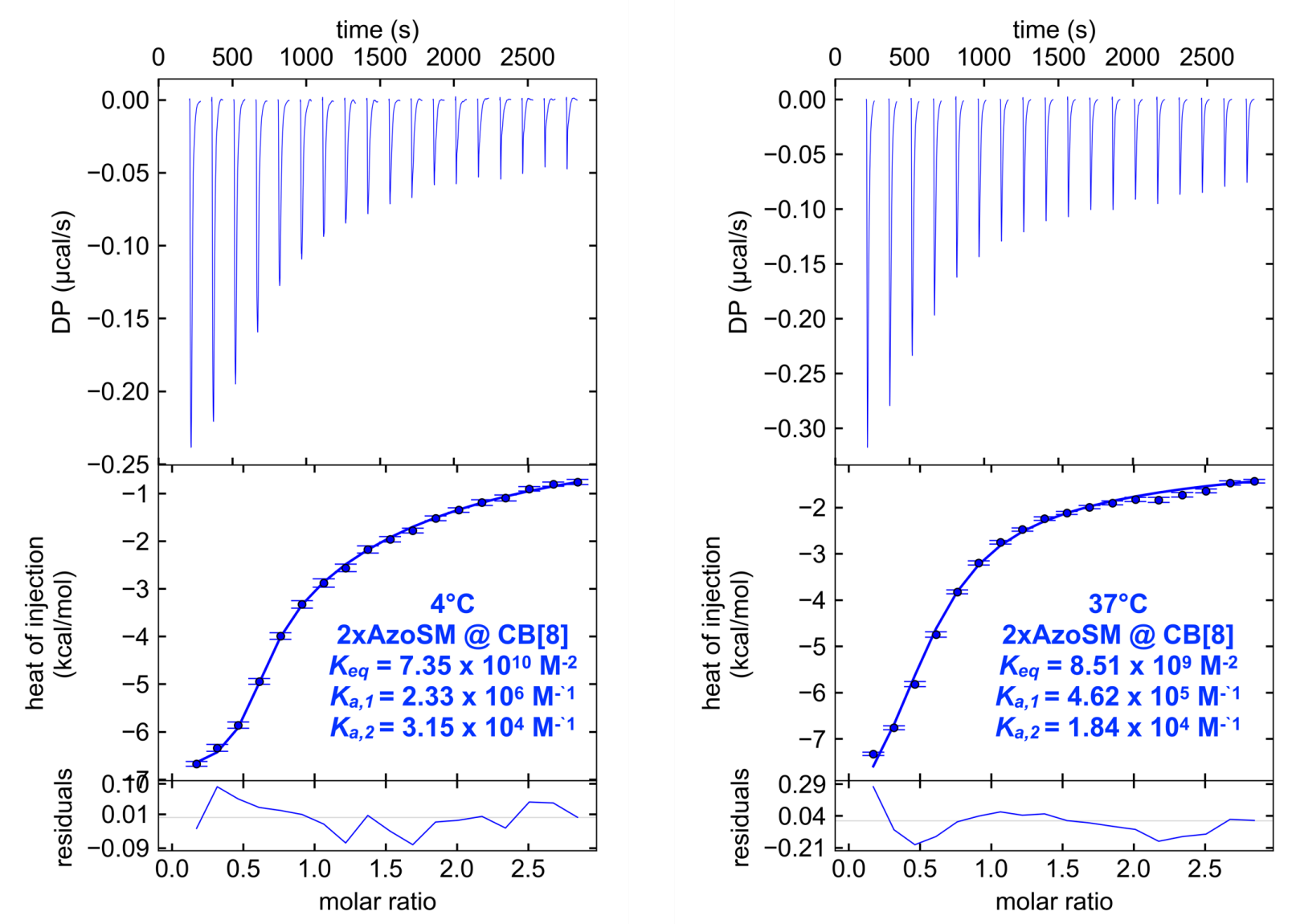

Figure S2: Supplemental ITC data at $4^{\circ} \mathrm{C}$ (left) and $37^{\circ} \mathrm{C}$ (right), with trans-AzoSM $(300 \mu \mathrm{M})$ titrated into $\mathrm{CB}[8](20 \mu \mathrm{M})$. Heats of injection from $\mathrm{n}=3$ separate experiments were fit simultaneously through global analysis to a sequential binding model, with residuals of the fit shown, to reveal the overall binding affinity $\left(K_{\text {eq }}\right)$ for the $\mathrm{CB}[8]-\mathrm{AzoSM}$ homoternary complex. 
Online Supporting Information

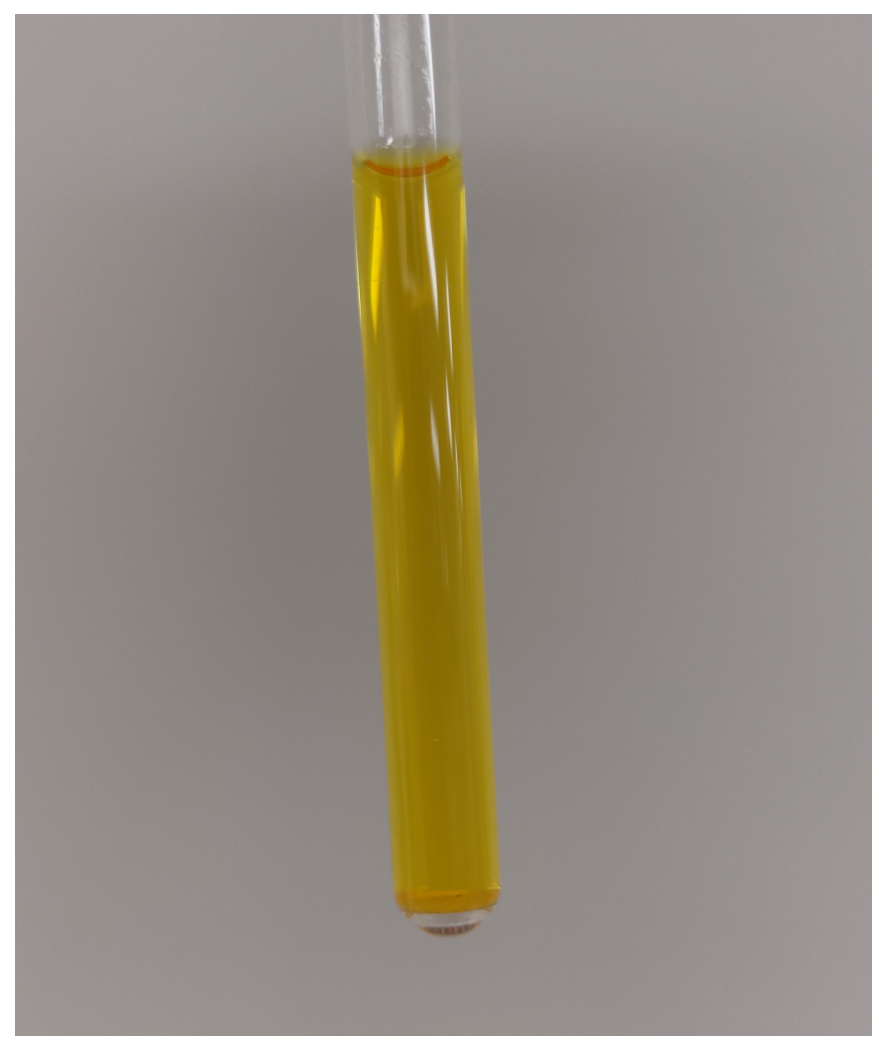

Figure S3: Photograph of NMR tube containing AzoSM and CB[8] immediately after UV irradiation, with evidence for a white $\boldsymbol{C B}[\mathbf{8}]$ precipitate upon photo-isomerization of AzoSM to its cis-form. 


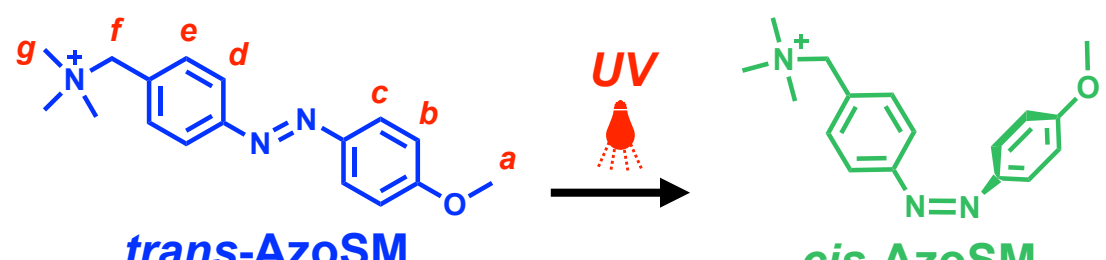

$$
*=\mathrm{CB}[8] \text { Protons }
$$

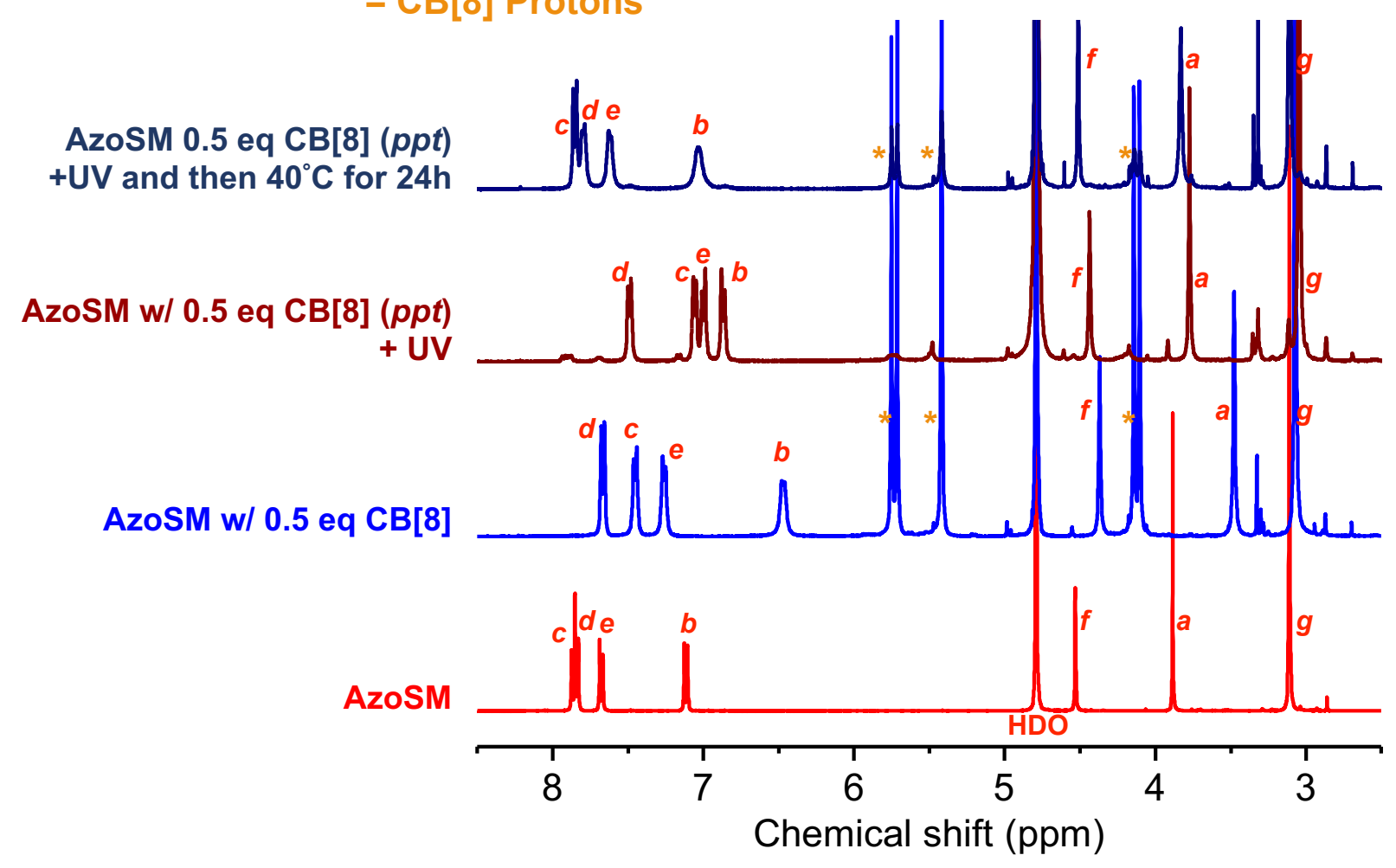

Figure S4: ${ }^{1} H-N M R$ characterization of AzoSM, highlighting the slow cis-to-trans thermal relaxation of this compound (top spectrum). This process necessitated 24 h of heating at $40{ }^{\circ} \mathrm{C}$, and while most $\boldsymbol{C B}[\mathbf{8}]$ remained precipitated trace soluble $C B[8]$ contributed to peak broadening of the reversed product relative to the pure trans-AzoSM. 

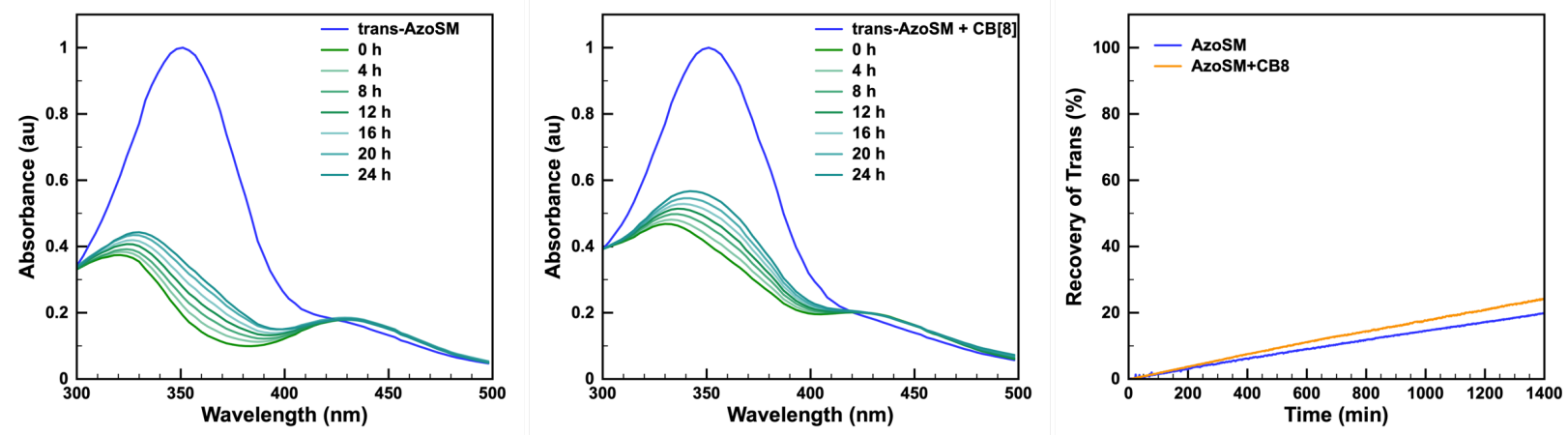

Figure S5: UV-vis spectroscopy of $0.15 \mathrm{mM} \mathrm{AzoSM}$ alone (left), or with the addition of 0.5 equivalents of $\boldsymbol{C B}[\mathbf{8}]$ (middle), assessing the room-temperature and dark recovery of the trans form after photoisomerization, where after $24 \mathrm{~h}$ (1440 minutes) $20 \%$ of the trans state was recovered without $\boldsymbol{C B}[\mathbf{8}]$, compared to $\sim 25 \%$ of the trans state with $\boldsymbol{C B}[8]$. 


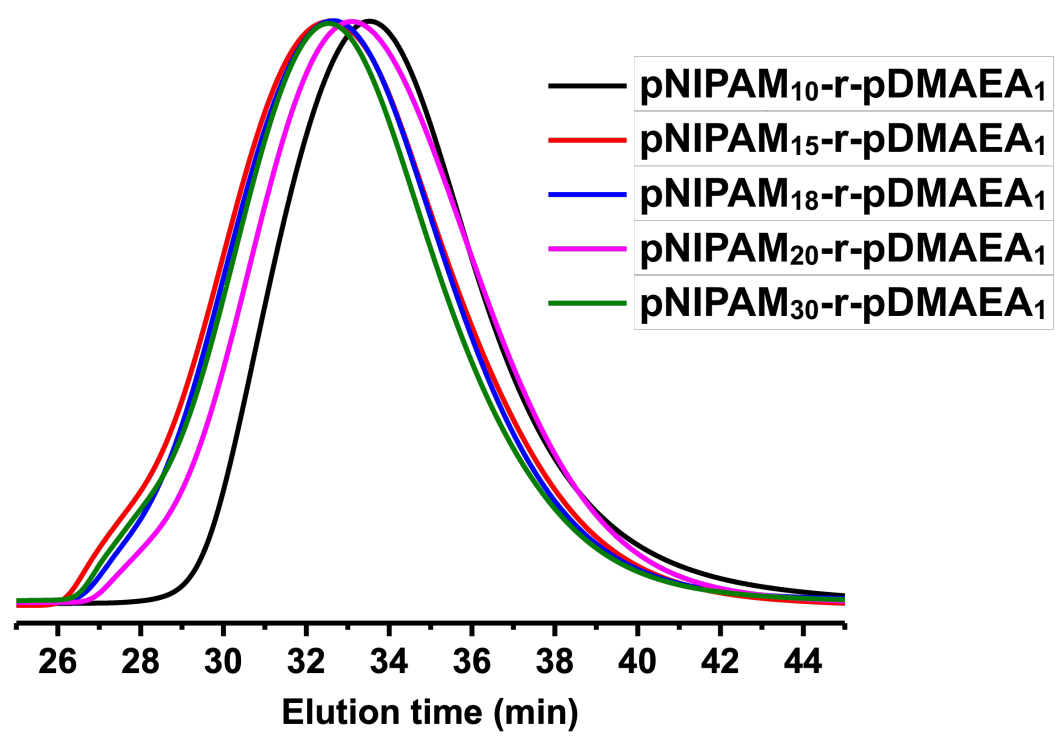

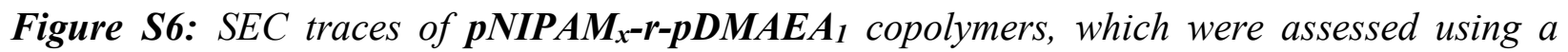
PEO standard to determine $M_{n}$. 


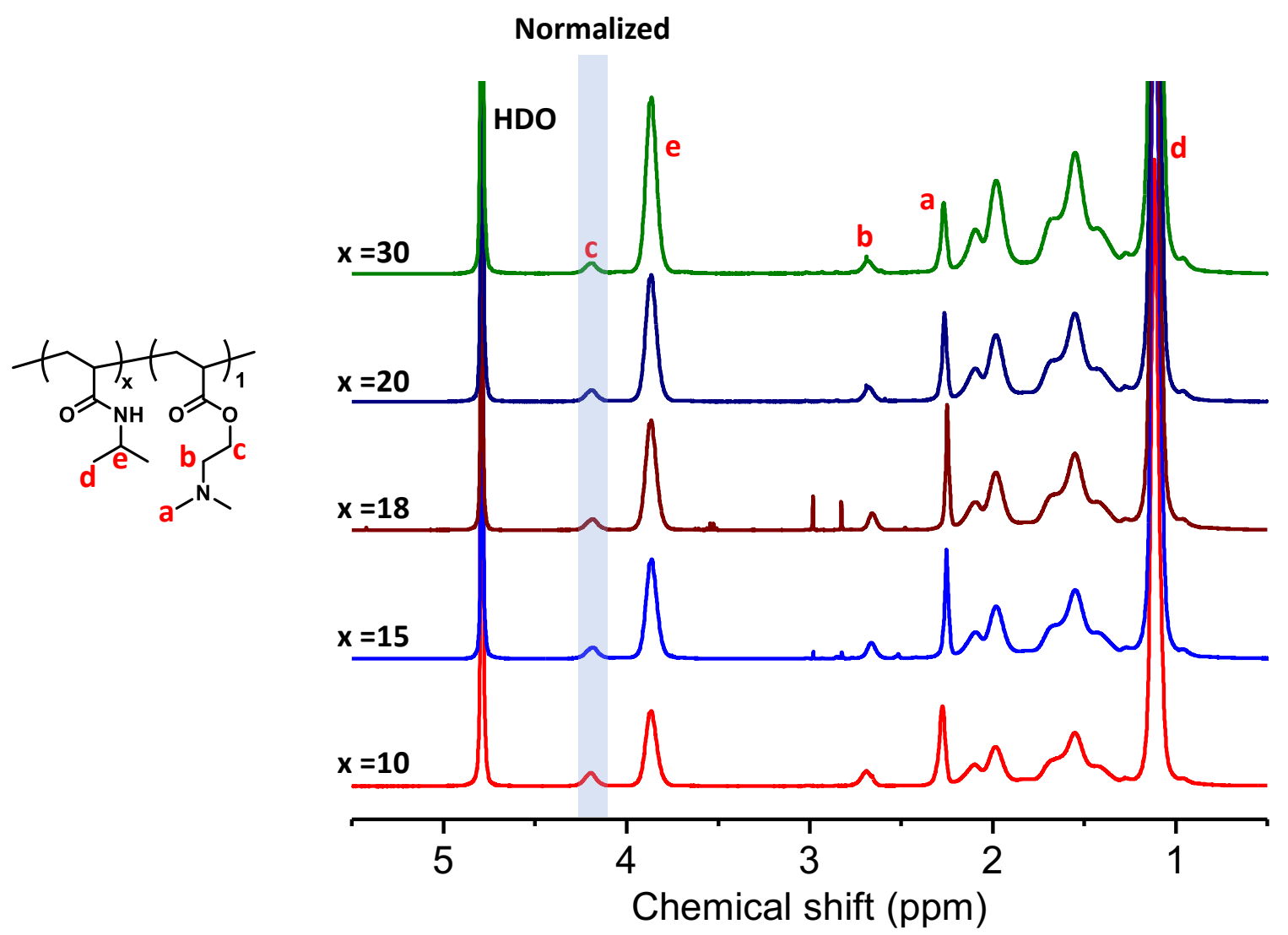

Figure S7: 'H-NMR characterization to determine monomer incorporation in synthesized pNIPAM -r-pDMAEA $\boldsymbol{A}_{1}$ copolymers. Spectra normalized to the DMAEA proton labelled with ' $\boldsymbol{c}$ ' and quantified relative to NIPAM proton labelled with ' $e$ '. 

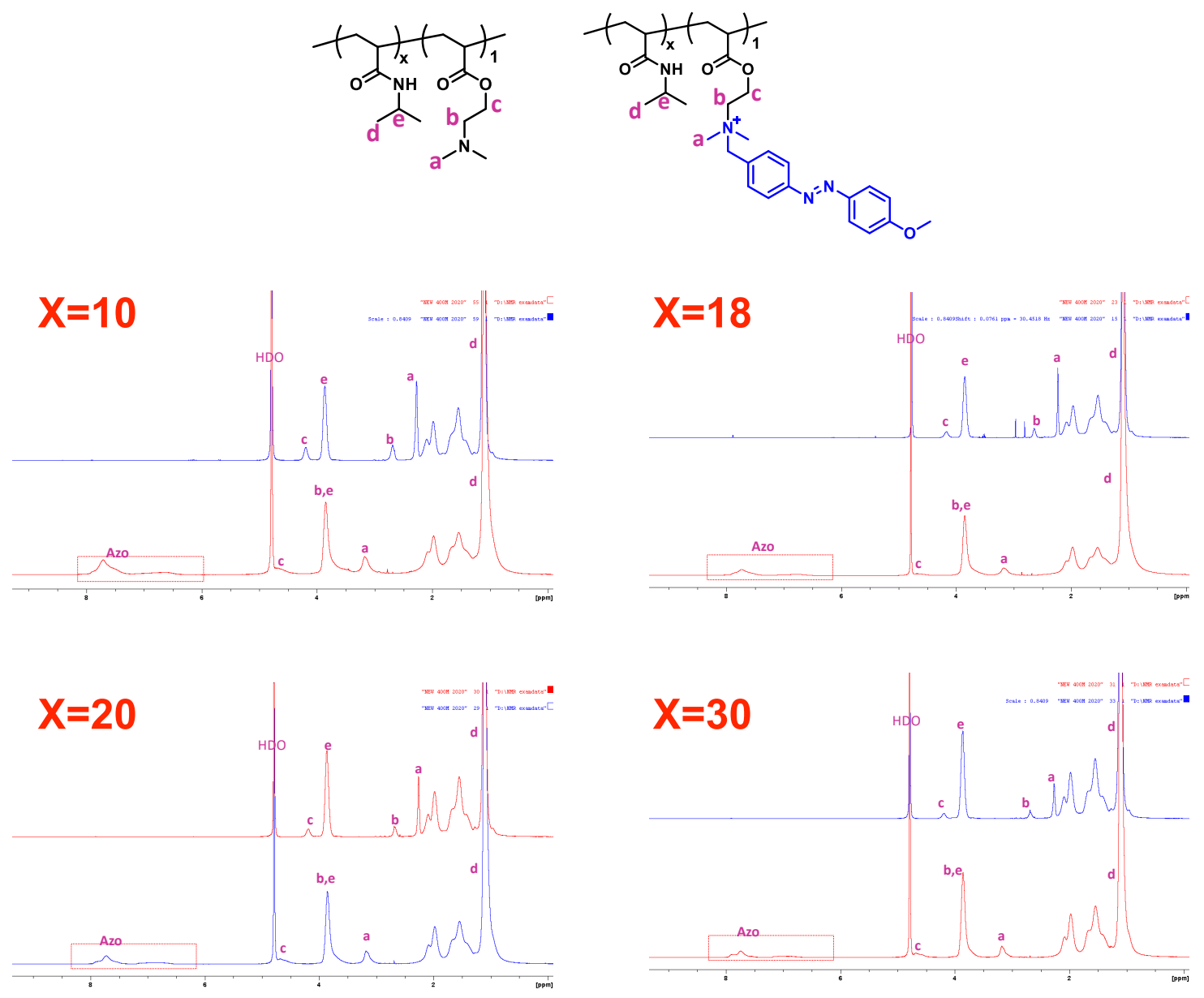

Figure S8: ${ }^{1} H-N M R$ characterization of to verify azobenzene modification of synthesized pNIPAM $\boldsymbol{M}_{\boldsymbol{x}}-\boldsymbol{r}$-pDMAEA $\boldsymbol{A}_{1}$ copolymers, where $X=10,18,20$, and 30. X=15 is shown in main text. 

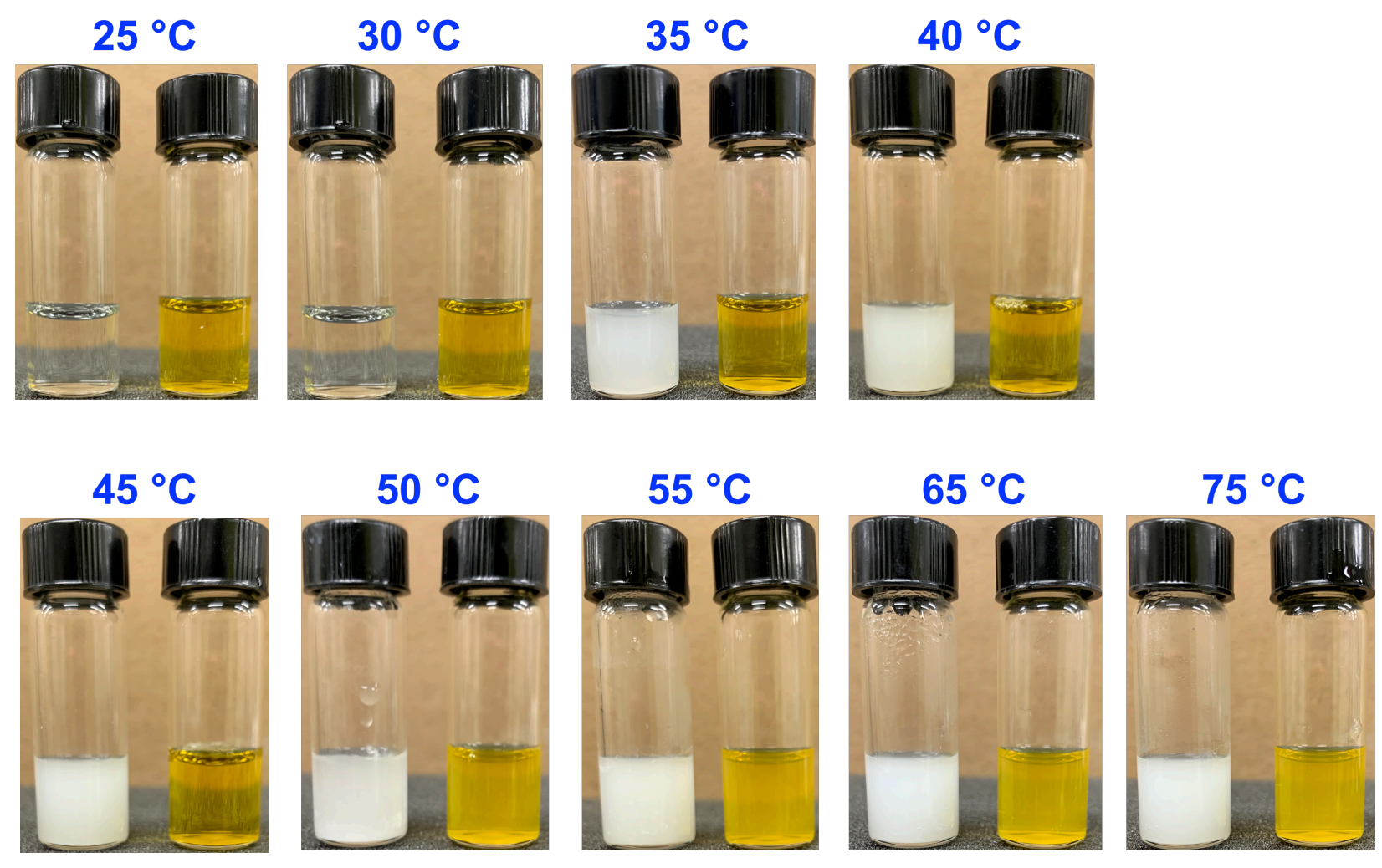

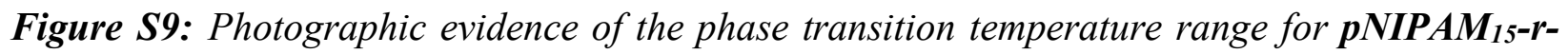

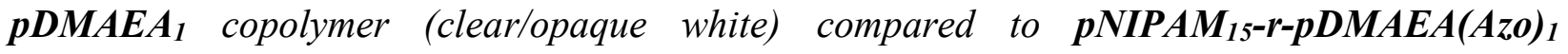
copolymer (yellow). 

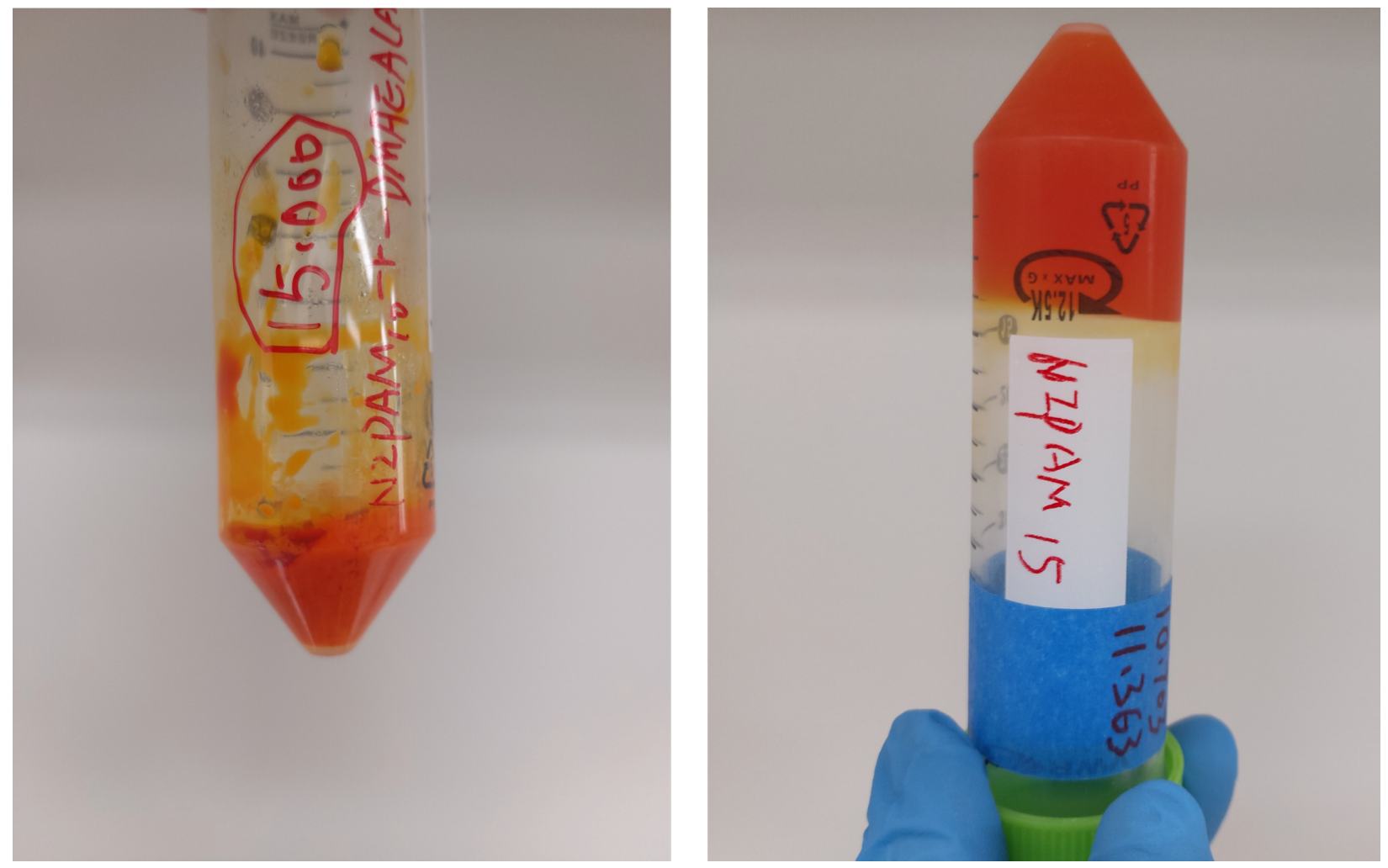

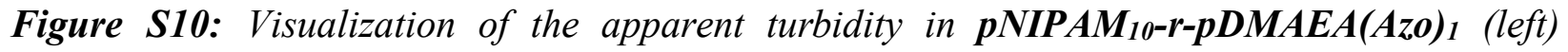
compared to pNIPAM $\mathbf{1 5}_{15}$-r-pDMAEA(Azo) ${ }_{1}$ (right) samples, even after a two month equilibration which attributed to incomplete solubility of $C B[8]$. 
Online Supporting Information

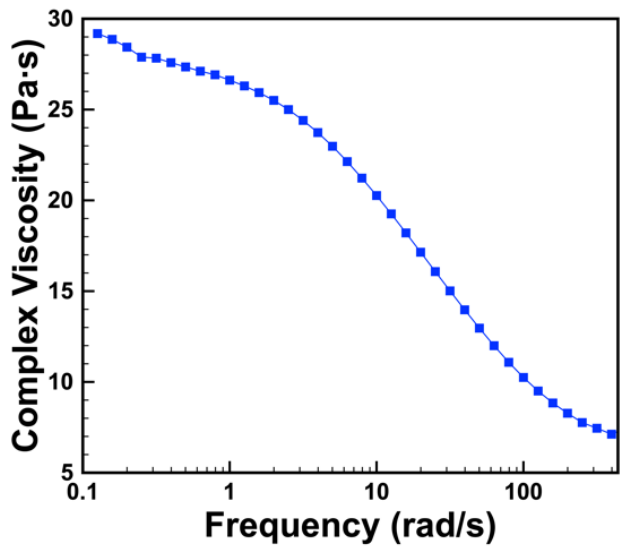

Figure S11: Complex viscosity measurements for pNIPAM $\mathbf{M}_{15}-\boldsymbol{r}-p D M A E A(A z o)_{1}$ in oscillatory rheology as a function of rate of oscillation. 

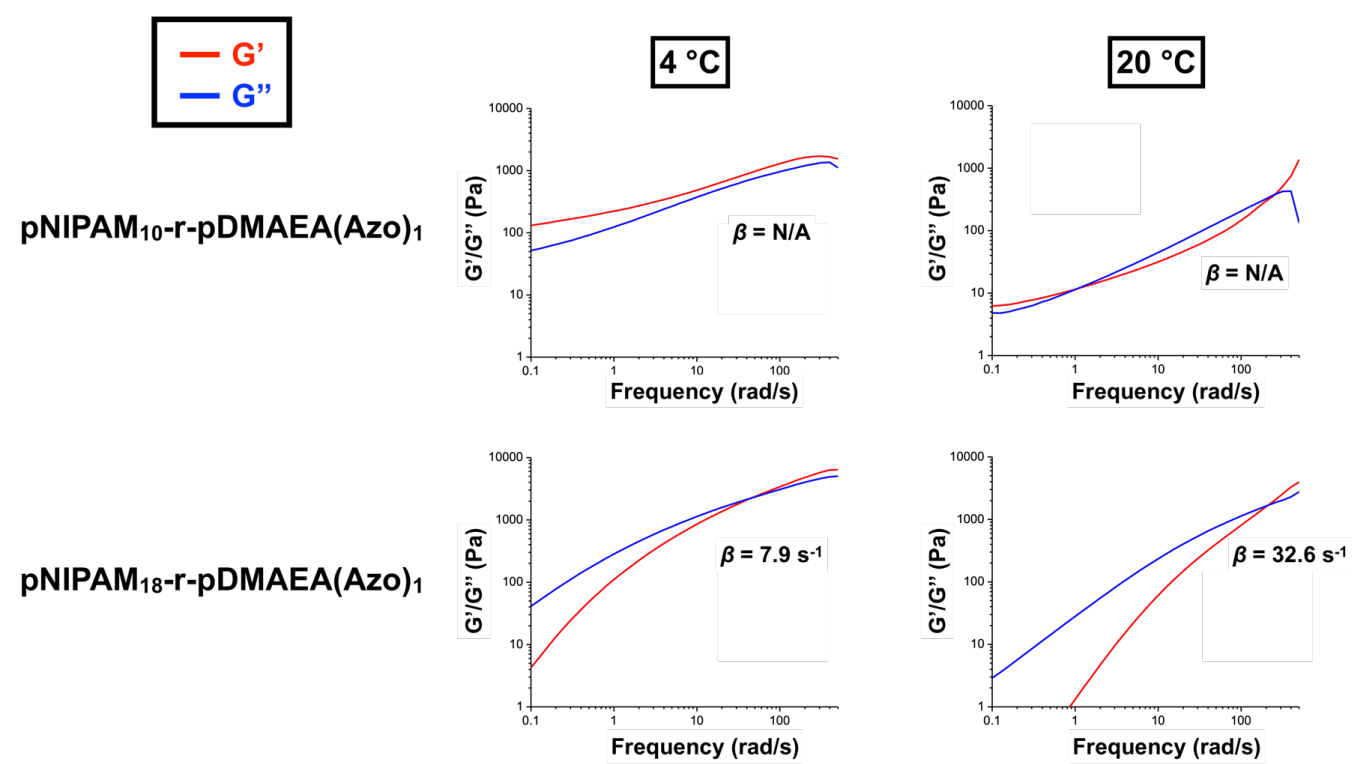

pNIPAM $_{20}$-r-pDMAEA(Azo) 1
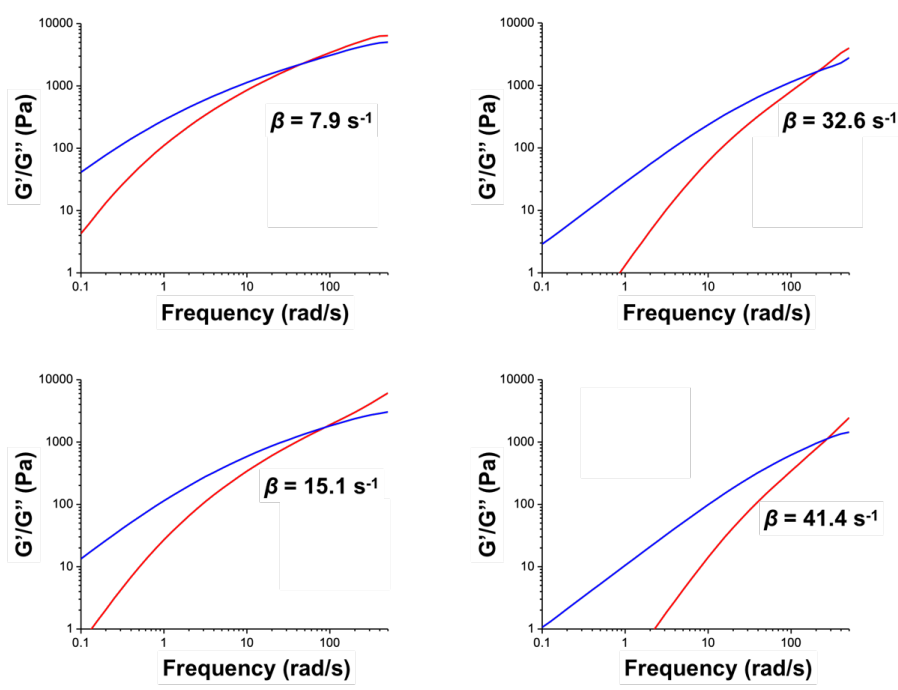

pNIPAM $_{30-r-p D M A E A(A z o)}{ }_{1}$
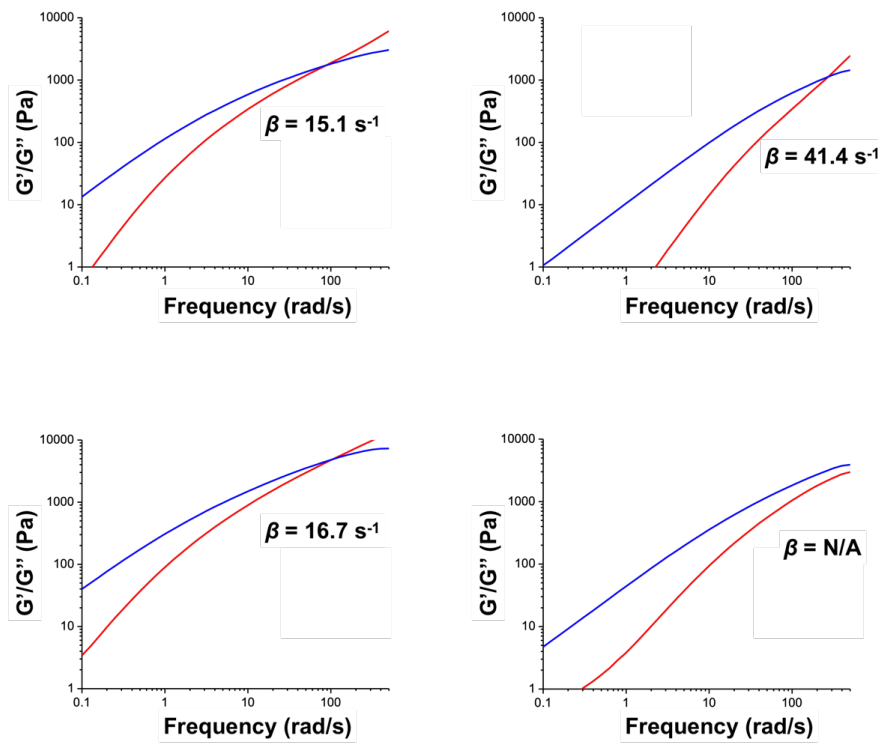

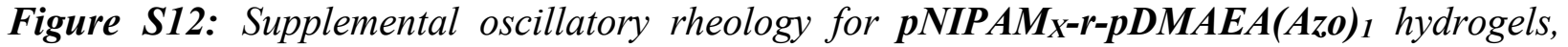
with $G^{\prime}$ (red) and $G^{\prime \prime}$ (blue) shown for copolymers with $X=10,18,20$, and 30 at $4^{\circ} \mathrm{C}$ (left) and $20^{\circ} \mathrm{C}(C)$. The $G^{\prime}-G^{\prime}$ ' crossover value, known as the bulk relaxation modulus $(\beta)$ is shown for samples where such a point is evident. 


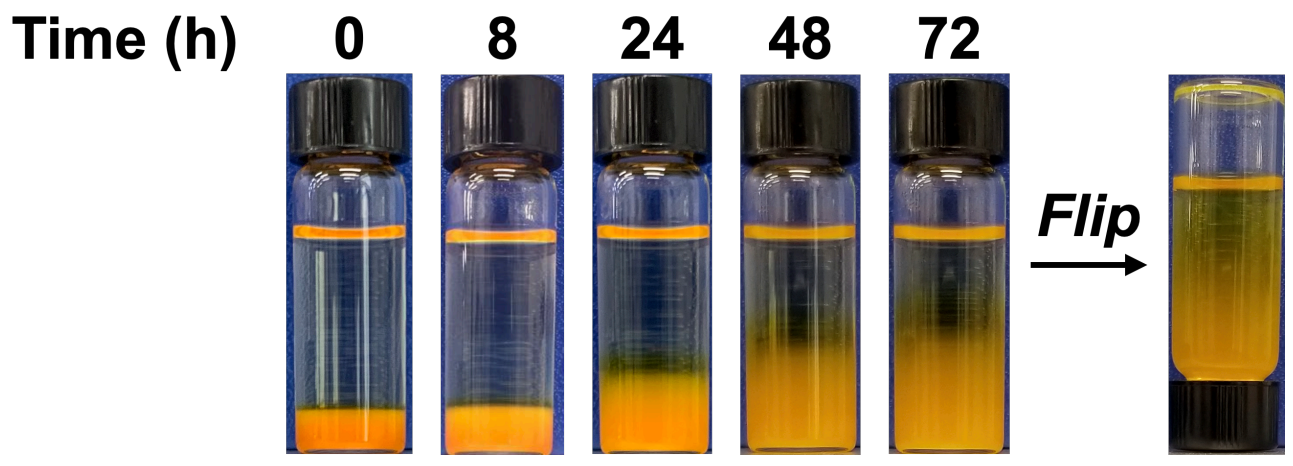

Figure S13: Swelling and erosion of 10 wt\% hydrogels prepared from pNIPAM $15-\boldsymbol{r}$ pDMAEA(Azo) ${ }_{1}$ with 0.5 equivalents of $\boldsymbol{C B}[\mathbf{8}]$. The gel structure progressively swelled and became eroded, and by $72 \mathrm{~h}$ had lost its self-supporting character as verified by vial inversion. 


\section{Online Supporting Information}
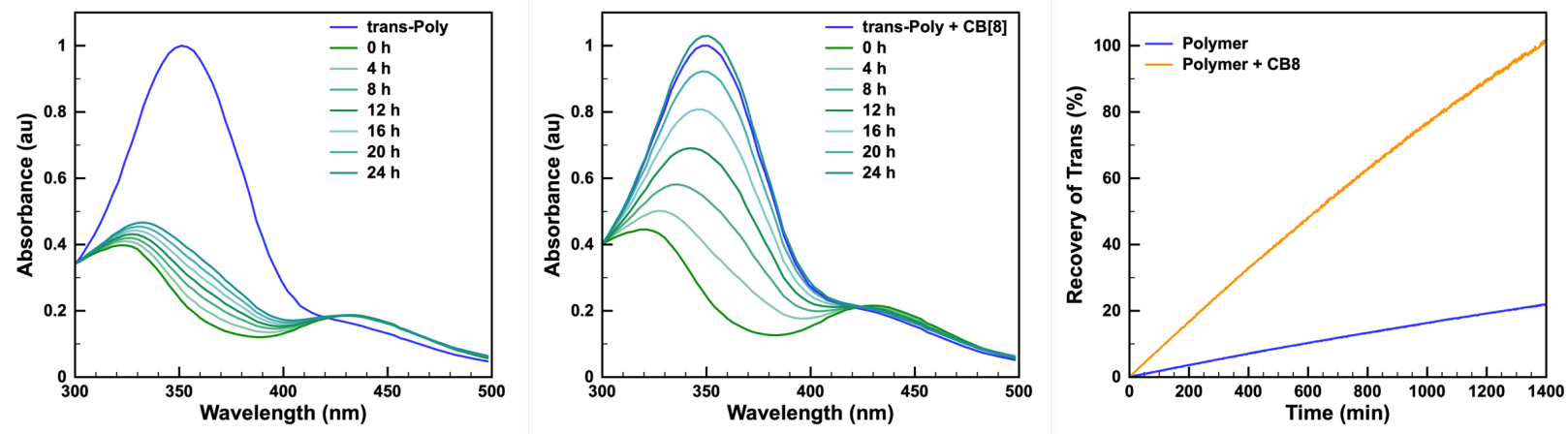

Figure S14: $U V$-vis spectroscopy of pNIPAM15-r-pDMAEA(Azo) $)_{1}(0.15 \mathrm{mM}$ of azobenzenes) alone (left), or with the addition of 0.5 equivalents of $\boldsymbol{C B}$ [8] (middle), assessing the roomtemperature and dark recovery of the trans form after photoisomerization, where after $24 h$ (1440 minutes) $\sim 23 \%$ of the trans state was recovered without $\boldsymbol{C B}[\mathbf{8}]$, compared to $\sim 100 \%$ of the trans state with $\mathbf{C B}[\mathbf{8}]$. 